\title{
GAIA Stillbirth Level of Diagnostic Certainty Terminology
}

National Cancer Institute

\section{Source}

National Cancer Institute. GAIA Stillbirth Level of Diagnostic Certainty Terminology. NCI

Thesaurus. Code C126856.

A subset of terminology related to stillbirth, developed by the Global Alignment of Immunization safety Assessment in pregnancy consortium to aid in monitoring and improving fetal and maternal outcomes. 